\title{
QUEEN'S
UNIVERSITY
BELFAST
}

\section{Calibration of BAS-TR image plate response to high energy (3-300 $\mathrm{MeV}$ ) carbon ions}

Doria, D., Kar, S., Ahmed, H., Alejo, A., Fernandez, J., Cerchez, M., Gray, R. J., Hanton, F., MacLellan, D. A., McKenna, P., Najmudin, Z., Neely, D., Romagnani, L., Ruiz, J. A., Sarri, G., Scullion, C., Streeter, M., Swantusch, M., Willi, O., ... Borghesi, M. (2015). Calibration of BAS-TR image plate response to high energy (3$300 \mathrm{MeV}$ ) carbon ions. Review of Scientific Instruments, 86(12), [123302]. https://doi.org/10.1063/1.4935582

\section{Published in:}

Review of Scientific Instruments

Document Version:

Publisher's PDF, also known as Version of record

\section{Queen's University Belfast - Research Portal:}

Link to publication record in Queen's University Belfast Research Portal

\footnotetext{
Publisher rights

Copyright (2015) AIP Publishing. This article may be downloaded for personal use only. Any other use requires prior permission of the author and AIP Publishing.

The following article appeared in Doria, D, Kar, S, Ahmed, H, Alejo, A, Fernandez, J, Cerchez, M, Gray, RJ, Hanton, F, MacLellan, DA, McKenna, P. Najmudin, Z, Neely, D, Romagnani, L, Ruiz, JA, Sarri, G, Scullion, C, Streeter, M, Swantusch, M, Willi, O, Zepf, M \& Borghesi, M 2015, 'Calibration of BAS-TR image plate response to high energy (3-300 MeV) carbon ions' Review of Scientific Instruments, vol 86, no. 12, 123302., and may be found at http://scitation.aip.org/content/aip/journal/rsi/86/12/10.1063/1.4935582

\section{General rights}

Copyright for the publications made accessible via the Queen's University Belfast Research Portal is retained by the author(s) and / or other copyright owners and it is a condition of accessing these publications that users recognise and abide by the legal requirements associated with these rights.
}

\section{Take down policy}

The Research Portal is Queen's institutional repository that provides access to Queen's research output. Every effort has been made to ensure that content in the Research Portal does not infringe any person's rights, or applicable UK laws. If you discover content in the Research Portal that you believe breaches copyright or violates any law, please contact openaccess@qub.ac.uk. 


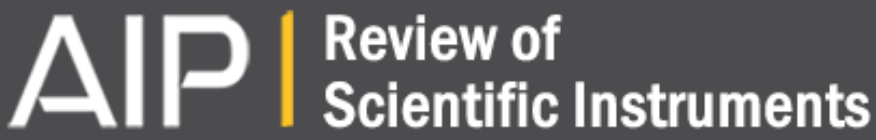

Calibration of BAS-TR image plate response to high energy (3-300 MeV) carbon ions

D. Doria, S. Kar, H. Ahmed, A. Alejo, J. Fernandez, M. Cerchez, R. J. Gray, F. Hanton, D. A. MacLellan, P. McKenna, Z. Najmudin, D. Neely, L. Romagnani, J. A. Ruiz, G. Sarri, C. Scullion, M. Streeter, M. Swantusch, O. Willi, M. Zepf, and M. Borghesi

Citation: Review of Scientific Instruments 86, 123302 (2015); doi: 10.1063/1.4935582

View online: http://dx.doi.org/10.1063/1.4935582

View Table of Contents: http://scitation.aip.org/content/aip/journal/rsi/86/12?ver=pdfcov

Published by the AIP Publishing

\section{Articles you may be interested in}

CR-39 track detector calibration for $\mathrm{H}, \mathrm{He}$, and $\mathrm{C}$ ions from 0.1-0.5 MeV up to $5 \mathrm{MeV}$ for laser-induced nuclear fusion product identification

Rev. Sci. Instrum. 86, 083307 (2015); 10.1063/1.4927684

Response functions of imaging plates to photons, electrons and 4 He particles Rev. Sci. Instrum. 84, 103510 (2013); 10.1063/1.4826084

Thomson spectrometer-microchannel plate assembly calibration for MeV-range positive and negative ions, and neutral atoms

Rev. Sci. Instrum. 84, 053302 (2013); 10.1063/1.4803670

Response functions of Fuji imaging plates to monoenergetic protons in the energy range 0.6-3.2 MeV Rev. Sci. Instrum. 84, 013508 (2013); 10.1063/1.4775719

Absolute calibration of photostimulable image plate detectors used as ( $0.5-20 \mathrm{MeV}$ ) high-energy proton detectors

Rev. Sci. Instrum. 79, 073301 (2008); 10.1063/1.2949388

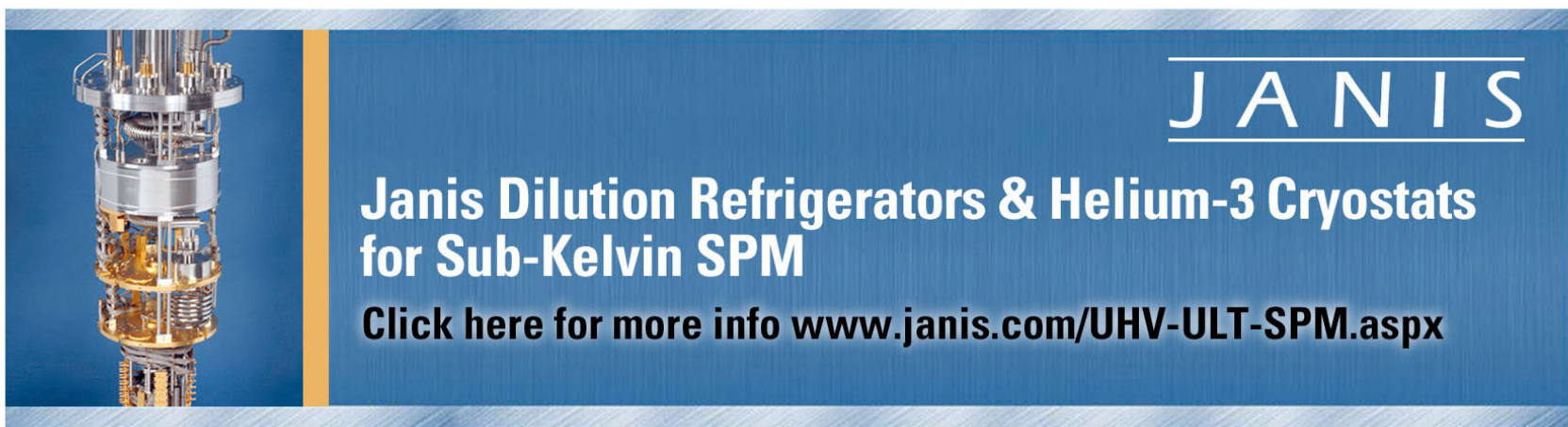




\title{
Calibration of BAS-TR image plate response to high energy (3-300 MeV) carbon ions
}

\author{
D. Doria, ${ }^{1}$ S. Kar, ${ }^{1, a)}$ H. Ahmed, ${ }^{1}$ A. Alejo, ${ }^{1}$ J. Fernandez, ${ }^{2,3}$ M. Cerchez, ${ }^{4}$ R. J. Gray, ${ }^{5}$ \\ F. Hanton, ${ }^{1}$ D. A. MacLellan, ${ }^{5}$ P. McKenna, ${ }^{5}$ Z. Najmudin, ${ }^{6}$ D. Neely, ${ }^{3}$ L. Romagnani, ${ }^{7}$ \\ J. A. Ruiz, ${ }^{8}$ G. Sarri, ${ }^{1}$ C. Scullion, ${ }^{1}$ M. Streeter, ${ }^{6}$ M. Swantusch, ${ }^{4}$ O. Willi, ${ }^{4}$ M. Zepf,${ }^{1,9}$ \\ and $\mathrm{M}$. Borghesi ${ }^{1,10}$ \\ ${ }^{1}$ Centre for Plasma Physics, School of Mathematics and Physics, Queen's University Belfast, \\ Belfast BT7 1NN, United Kingdom \\ ${ }^{2}$ Instituto de Fusión Nuclear, Universidad Politécnica de Madrid, Madrid 28006, Spain \\ ${ }^{3}$ Central Laser Facility, Rutherford Appleton Laboratory, Didcot, Oxfordshire OX11 OQX, United Kingdom \\ ${ }_{4}^{4}$ Institut für Laser-und Plasmaphysik, Heinrich-Heine-Universität, Düsseldorf 40225, Germany \\ ${ }^{5}$ Department of Physics, SUPA, University of Strathclyde, Glasgow G4 ONG, United Kingdom \\ ${ }^{6} \mathrm{John}$ Adams Institute for Accelerator Science, The Blackett Laboratory, Imperial College, \\ London SW7 2BW, United Kingdom \\ ${ }^{7}$ LULI, Ecole Polytechnique, CNRS, Route de Saclay, Palaiseau Cedex 91128, France \\ ${ }^{8}$ Colegio Los Naranjos, Fuenlabrada, Madrid 28941, Spain \\ ${ }^{9}$ Helmholtz Institut Jena, Jena D-07743, Germany \\ ${ }^{10}$ Institute of Physics of the ASCR, ELI-Beamlines Project, Na Slovance 2, Prague 18221, Czech Republic
}

(Received 25 May 2015; accepted 31 October 2015; published online 15 December 2015)

\begin{abstract}
The paper presents the calibration of Fuji BAS-TR image plate (IP) response to high energy carbon ions of different charge states by employing an intense laser-driven ion source, which allowed access to carbon energies up to $270 \mathrm{MeV}$. The calibration method consists of employing a Thomson parabola spectrometer to separate and spectrally resolve different ion species, and a slotted CR-39 solid state detector overlayed onto an image plate for an absolute calibration of the IP signal. An empirical response function was obtained which can be reasonably extrapolated to higher ion energies. The experimental data also show that the IP response is independent of ion charge states. (C) 2015 AIP Publishing LLC. [http://dx.doi.org/10.1063/1.4935582]
\end{abstract}

\section{INTRODUCTION}

A wide range of passive detectors has been developed to diagnose different types of ions, such as solid state detectors (e.g. CR-39-a plastic polymer that is used as a nuclear track detector), radiochromic films (RCF) which have a self-developing active medium consisting of an emulsion, scintillators, and image plates (IPs). ${ }^{1}$ Scintillators and image plates are based on the principle of photoluminescence (PL), which occurs when an atom is excited to a higher energy state followed by its return to a lower state while emitting a photon. PL is characterised by two forms of luminescence: fluorescence and phosphorescence. While scintillators are made of fluorescent material (spontaneously releasing the stored energy in a short time, typically less than microseconds), the IP is made of phosphors, and owing to its phosphorescent properties, it can store the energy for a long time until being stimulated by photons of suitable wavelength. ${ }^{2-4}$ The image plate was developed by Fuji Photo Film Co., Ltd. in early 1980s and since then it has been a popular film detector in physics and medicine ${ }^{4}$ due to attractive properties such as high spatial resolution (up to $10 \mu \mathrm{m}$ ), high dynamic range (4-5 orders of magnitude), and a non-disposable nature, as the signal on the IP can be erased after every use.

a)Electronic mail: s.kar@qub.ac.uk
Image plates are currently being widely used in laser plasma experiments for detection of charged particles (electrons and ions) and X-rays due to their passive nature and aforementioned advantages. The phosphorescence mechanism results in a fairly linear response of IP to the energy deposited by the ionizing radiation. ${ }^{5}$ However, the signal on IP will vary depending on the type of radiation as well as its energy. Commonly used image plate types, such as BAS-MS and BAS$\mathrm{TR}$, have been calibrated for electrons, ${ }^{6-8} \mathrm{X}$-rays ${ }^{5,9}$ and a few ion species of low atomic mass, such as $\mathrm{H}, \mathrm{D}$, and He. ${ }^{10-15}$ Amongst heavier ion species, carbon ions occupy a particularly important position, e.g. in light of their application in cancer therapy ${ }^{16,17}$ Furthermore, in current research aimed to develop novel accelerator concepts based on laser drivers, ${ }^{18}$ carbon ions are often the key species enabling the characterization of specific acceleration mechanisms. ${ }^{19-21}$ As image plates are often the detector of choice for the spectral characterization of the carbon ions in these experiments, their absolute calibration to this ion species is therefore crucial and timely.

In this paper, we report for the first time the calibration of BAS-TR type of IP for different charge states of carbon ions spanning over a wide range of energy up to $270 \mathrm{MeV}$. BAS-TR is the commonly used image plate for ion detection in laser plasma experiments due to its bare phosphor layer, which allows detection of low energy ions. The calibration was obtained by using slotted CR-39 nuclear track detector over the IP, which enabled direct comparison between the 
signal on IP and the absolute number of particles given by the number of tracks on CR-39. The calibration was obtained separately for different charge states of carbon ions, which suggested that the IP response is independent of the charge state of ions. The structure of the remainder of the paper is as follows. Section II briefly explains the experimental setup and methods used to obtain the data in the experiment. Section III discusses the mechanism of particle detection in the two types of detectors (CR-39 and IP) used in the calibration, which is followed by the presentation of the calibration method, results and discussion in Section IV.

\section{EXPERIMENTAL SETUP}

The experiment was carried out at the Rutherford Appleton Laboratory (RAL), STFC, UK, by employing the Petawatt arm of the VULCAN laser system. The laser operates in the infrared range at a wavelength of $1053 \mathrm{~nm}$. The high power of the laser pulse is achieved by employing the chirped pulse amplification (CPA) technique, which provides short pulses sub-picosecond ( $\sim 750 \mathrm{fs}$ ) duration with pulse energy typically around $500 \mathrm{~J}$ before the compression. In this experiment, the laser beam was focused, at normal incidence, on ultra-thin (sub-micron) foils of amorphous carbon and gold by using an $f / 3$ off-axis parabolic mirror. A single plasma mirror was used before the target in order to suppress pre-pulses and Amplified Spontaneous Emission (ASE) associated with the laser beam prior to the arrival of the intense short pulse on the target. ${ }^{22-24}$ Taking into account the energy losses in the compressor gratings and the plasma mirror, the peak laser intensity on target was typically of the order of $10^{20} \mathrm{~W} \mathrm{~cm}^{-2}$.

The energetic ion beam produced by the interaction of such pulses with solid foils is inherently multispecies-in addition to the ion species belonging to the target bulk, hydrocarbon contaminants present on both sides of the foil targets provide a rich source of hydrogen and carbon ions. ${ }^{25,26}$ In particular, the use of ultrathin foils allows access to volumetric acceleration scenarios, ${ }^{18}$ where heavier ions such as carbon can be accelerated with a comparable efficiency to protons (in contrast to TNSA acceleration from thicker targets where acceleration of protons is strongly favoured). A high resolution Thomson Parabola Spectrometer (TPS) was employed in order to discriminate between different ion species while dispersing the ions according to their kinetic energy. ${ }^{27,28}$ As shown in Fig. 1(a), the TPS was mounted along the laser axis and placed at the rear side of the target at a distance of $1.2 \mathrm{~m}$ from the target surface, for having a suitable ion flux at the detector. A strong magnetic field (1 T) and large drift distance $(30 \mathrm{~cm})$ were used to achieve high energy resolution $(\mathrm{E} / \delta \mathrm{E} \sim 65$ at $270 \mathrm{MeV})$. An electric field of the order of $20 \mathrm{kV} / \mathrm{cm}$ was applied over a $15 \mathrm{~cm}$ long region in order to achieve ion species separation at the high energy end of the ion spectrum. ${ }^{28}$ An IP BAS-TR and a slotted CR-39 detectors were used in a stack configuration as detector for the TPS, ${ }^{15,29,30}$ as shown in Fig. 1(b), in order to cross-calibrate the IP signal with the absolute number of ions obtained by counting the pits in CR-39. The image plates were wrapped with $6 \mu \mathrm{m} \mathrm{Al}$ foil in order to avoid their exposure to ambient light before scanning.
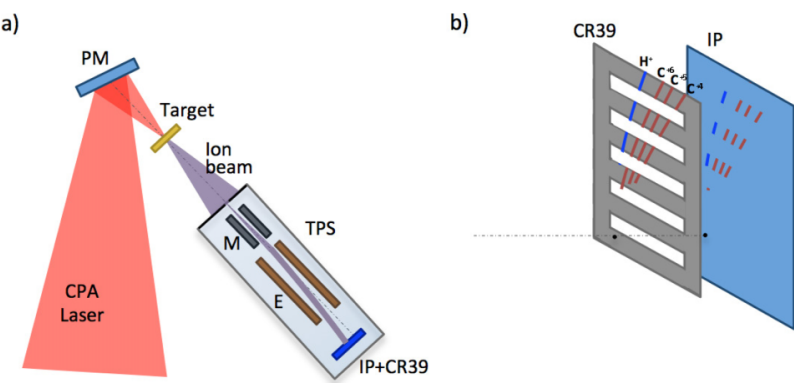

FIG. 1. (a) Layout of the experimental setup: the TPS consists of a magnetic dipole M, electric field plates E and detector, CR-39+IP. (b) Design of the detector assembly (CR-39+IP) used for the IP calibration: it consists of one slotted CR-39 with periodic slot size of $1 \mathrm{~mm}$ and one IP BAS-TR behind it placed in direct contact.

\section{PARTICLE REGISTRATION AND DETECTION IN IP AND CR-39}

\section{A. BAS-TR image plate}

Among several types of IPs, the BAS-TR was selected due to the absence of the plastic protective layer above the phosphor layer, which is usually present in other types of IP. ${ }^{2,9}$ Although the protective layer helps preserving the efficiency of the phosphor, due to the hygroscopic nature of the phosphor, it prevents detection of ions which are not energetic enough to cross the layer. For instance, the $10 \mu \mathrm{m}$ thick protective layer typically present in other types of IPs is sufficient to stop carbon ions with energy up to $10 \mathrm{MeV}$. BAS-TR contains a $50 \mu \mathrm{m}$ thick active layer, consisting of photo-stimulable crystal grains $\left(\mathrm{BaFX}: \mathrm{Eu}^{2+}\right.$, where $\left.\mathrm{X}=\mathrm{Cl}, \mathrm{Br}\right)$ of average density $5.2 \mathrm{~g} / \mathrm{cm}^{3}$ and $5 \mu \mathrm{m}$ in size, embedded in a urethane resin. ${ }^{2,4}$ The half-life for spontaneous decay from its metastable states is typically of the order of days, following a faster decay within the first $30 \mathrm{~min}$ after exposure. ${ }^{3,10,15}$ Therefore, the energy stored in the IP can be retrieved by stimulating the excited metastable state, even several hours after the exposure. The stimulation can be done by optical photons in the red part of the visible spectrum, and the energy is released as UV light referred to as photo-stimulated luminescence (PSL). Using a red laser diode of $650 \mathrm{~nm}$ wavelength for de-excitation of the metastable states and a PMT to read the emitted UV photons, commercially available image plate scanners provide high resolution 2D images of the signal stored across the IP with a pixel size down to $10 \mu \mathrm{m}$ and a 16-bit depth in grayscale. After scanning, the IP can be reused by erasing any remaining signal in the IP through exposure of the phosphor to bright white light for approximately $15 \mathrm{~min}$.

For the data obtained in our experiment, the IPs were scanned between 1-2 $\mathrm{h}$ after the exposure, due to the time required for completing the shot cycle and retrieving the IPs from the interaction chamber. The image plates were scanned using the Fuji FLA-5000 scanner with a Resolution (R) of 50 (i.e., $50 \mu \mathrm{m} \times 50 \mu \mathrm{m}$ square pixel size); dynamic range or Latitude (L) of 5; Sensitivity (S) of 5000; and 16-bit depth (G). 2,15,31 The PSL stored in each pixel of the 16-bit TIFF output image can be obtained by converting its bit value (QL) using the formula provided by Fuji, ${ }^{15,31}$

$$
P S L=\left(\frac{R}{100}\right)^{2} \times \frac{4000}{S} \times 10^{L \times\left(\frac{Q L}{2 G_{-1}}-\frac{1}{2}\right)} .
$$




\section{B. CR-39 Nuclear track detector}

The CR-39 is a plastic polymer used as a solid-state track detector in nuclear science to detect ions and neutrons. ${ }^{32-35}$ The main advantage of CR-39 is its insensitiveness to photonic radiation. Due to their electronic energy loss, ions leave trails of damaged polymer as they travel through the CR-39, eventually slowing down to rest. Since the damaged polymer has a faster etching rate than the normal CR-39 plastic, the ion trails develop into cone shaped pits upon etching the CR-39 in alkali solution.

The sheets of CR-39 used in the experiment were $1 \mathrm{~mm}$ thick and of $1.3 \mathrm{~g} / \mathrm{cm}^{3}$ density. The tracks left in the bulk were revealed by etching the CR-39 in $6 \mathrm{M}$ sodium hydroxide solution at $85^{\circ} \mathrm{C}$ for a few tens of minutes in order to enlarge the pits of carbon ions enough to make them clear and distinguishable under microscope analysis. Images of the etched CR-39 were grabbed by an 8 bit CCD camera using Scion plug-in for Image $\mathrm{J}^{36}$ and adequate magnification; the typical pit density was of the order of $100 \mathrm{ions} / \mathrm{mm}^{2}$ and therefore the pits could be counted with less than a few percent error. $^{37,38}$

\section{CALIBRATION OF IP RESPONSE FOR C IONS}

\section{A. Method}

In the TPS diagnostic, ions with different charge to mass ratio are dispersed on the detector plane along distinctive parabolas having the same origin. By measuring the field strengths and accounting for the geometrical layout of the TPS, it is possible to link precisely the particle position on the detector with its kinetic energy by means of an analytical formula. ${ }^{15,28}$ Based on this principle, the raw IP data (see Fig. 2, for example) were analysed by using a MATLAB code, as discussed below, in order to obtain the ion spectra in terms of PSL versus ion energy.

By using a graphical user interface, a Region-of-Interest (ROI), for example, a specific parabolic trace, was selected in the Matlab code to process the data for a given ion species. The first step was to correct the signal in the ROI by subtracting the background in the IP generated by high energy X-rays and scattered ions from the pinhole. Due to the non-uniform background level across the detector (see Fig. 2), the signal profile along the ROI was corrected by subtracting the average of the background profiles collected within boxes having same size as the ROI, on either sides of the ion trace as shown in

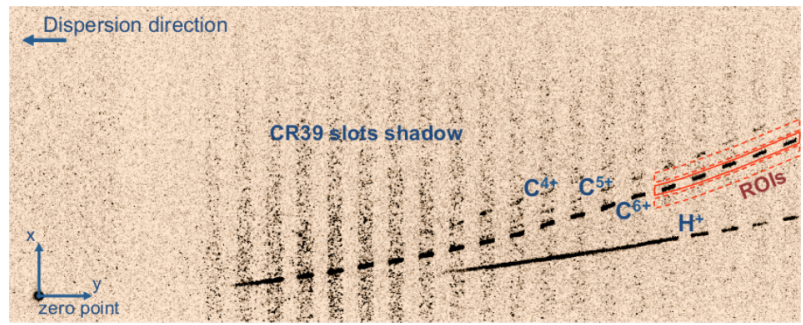

FIG. 2. IP scan image showing the shadow of the CR-39 slots and the signals of proton and carbon ions. The red contours surrounding the $\mathrm{C}^{6+}$ TPS trace are an example of ROIs used in signal and background analysis.
Fig. 2. After identifying the Z/A (charge-to-mass) ratio of the ion species corresponding to the trace in the ROI, the Y co-ordinate (energy dispersion axis) of the ROI was converted into ion energy by using Equation (1) in Ref. 28. Finally, the amplitude of the signal for each Y co-ordinate was obtained by integration of the signal in the direction orthogonal to the dispersion direction, i.e., all the pixels in the ROI having the same Y co-ordinate. Each data point in the resulting spectra now corresponds to the PSL signal on the IP caused by the ions within a small energy range defined by the width of a pixel (i.e., $50 \mu \mathrm{m}$ in our case) and the location of the pixel along the Y-axis.

The shadow of the CR-39 bar is clearly visible in the IP scanned image (Fig. 2), causing discontinuities in the ion traces, and consequently in the ion spectra obtained through the procedure described above, where each discontinuity represents an edge of a CR-39 slot. Therefore, the IP response can be obtained, in principle, by correlating the PSL signal near the edge of a discontinuity with the number of particles obtained from the adjacent CR-39 bar. This is necessary as the CR-39 particle tracks and the PSL cannot be measured at the same time, since the particles are generally stopped in the CR-39 (1 mm-thick CR-39 is able to stop carbon ions with energy up to $230 \mathrm{MeV}) .{ }^{39}$ In order to obtain a PSL calibration, the CR-39 data inside the ROI were processed in a similar way as the IP data, i.e., a lineout for number of pits along the Y-axis was obtained by binning the ROI orthogonal to the Y-axis by the same Y-step size of $50 \mu \mathrm{m}$. In this way, it was possible to overlap the ion spectra obtained from IP and CR-39 in order to obtain a correlation between PSL and number of particles at each edge of the CR-39 slots.

The fundamental assumption behind the calibration procedure is that the PSL and CR-39 signals should be matched to form a continuous profile, as they represent the same spectrum. Therefore, a calibration factor (PSL/ion) at each edge of the CR-39 slots, corresponding to a given ion energy, can be obtained by matching the PSL signal with the CR-39 pit density as shown in Fig. 3. The factor is obtained

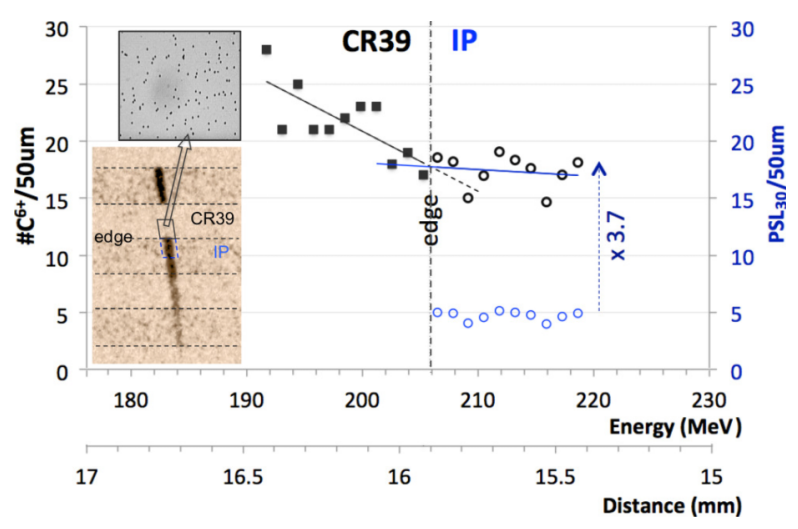

FIG. 3. The graph shows the section of the edge of the stack detector (i.e., the IP on the right-hand side and the CR-39 on the left-hand side, separated by the vertical dashed line) highlighted in the inset on the left bottom corner. The blue empty circles represent the IP signal rescaled to $30 \mathrm{~min}$ after exposure and the black solid squares represented the CR-39 pits signal (also shown as the inset at the left top corner). The black empty circles represent the IP signal rescaled to match the CR-39 signal at the edge. The rescaling factor gives the IP response. 
by approximating the CR-39 and PSL signals around the edge by straight lines and scaling the PSL to create a continuous profile. For the case shown in Fig. 3, the IP signal (represented by the blue empty circles) was multiplied by a factor 3.7 in order to match the CR-39 signal (black solid squares). The IP response is therefore the inverse of this factor, which is $0.27 \mathrm{PSL} / \mathrm{\#}^{6+}$ for ion energy of $206 \mathrm{MeV}$. The estimation of the ion energy at the IP surface took into account the energy reduction due to the $6 \mu \mathrm{m}$ thick Al filter placed in front of the IP; this correction is important for carbon ions with energy up to few 10's of MeV. The corresponding PSL signal in the track however is not affected by the presence of the Al filter, because (1) secondary ionising radiation produced by the ions is negligible for these energies, and (2) ions do not spread out by scattering as the thin filter was in contact with the IP. The correlation between PSL and adjacent pits density profiles can be done with very good accuracy provided that the flux of particles varies slowly along the TPS trace. The uncertainty associated with the fluctuation of the signal around the edge of the CR-39 slot was evaluated and experimental error was estimated.

Ions with energy above a certain threshold, which depends on the ion species, can traverse the CR-39 and reach the IP. For these ions, the TPS trace recorded by the IP is continuous across the CR-39 slots, as can be seen towards the high energy end of the carbon trace in Fig. 2, which is also shown as a zoomed image in Fig. 3. In this case, a direct comparison is possible between the number of pits observed in a CR-39 bar and the PSL signal obtained behind it. However, the IP response evaluated in this region will correspond to the lower kinetic energy possessed by the ions while impacting on the IP surface, which can be calculated considering the CR-39 thickness and the $6 \mu \mathrm{m} \mathrm{Al} \mathrm{filter.}$

\section{B. Uncertainties and error analysis}

In the evaluation of the IP response to ions, a number of factors has to be taken into account in order to evaluate the uncertainty associated with the measurement. Some factors arise from the characteristics of the IP itself and others from the calibration method. The method used in this paper introduces a relative error in the measurement of about $15 \%$, which is directly associated to the fluctuation of the data points about the straight-line fit, as can be seen in Fig. 3. Regarding the IP, the main factors to address are (1) the fading of the signal stored in the active layer, (2) the intensity of the diode laser that stimulates the IP phosphor, (3) the calibration of the PMT of the scanner that reads the photo-stimulated light coming from the IP, and (4) the surface quality of the phosphor layer. ${ }^{40}$ All these factors can lead to differences in the PSL output for the same input signal. The issues related to the scanner ((2) and (3)) can be mitigated by calibrating the scanner with the calibration tools provided by Fuji Film, while concerning the phosphor layer, it is recommended to use an IP that has a good quality surface without any scratches and stains.

The factor that gives the largest variation in the PSL signal is the fading of IP signal due to spontaneous decay of the metastable states of the phosphor. A good detector of this type should be able to hold the information for tens of minutes or hours exhibiting only a small variation in the intensity of the signal when read. However, as long as the variation of the signal with time due to spontaneous emission is characterised, the procedure to estimate the ion flux at a given energy from the PSL can be standardised.

In the literature many papers describe fading curves obtained with different techniques and ionizing radiation, but the curves from these papers differ significantly at the earlier decay stage, within a few minutes from the exposure. . $^{3,10,15,41}$ A reason may be that, since the calibrations are generally done using ionizing radiation (e.g., photon, alpha) produced by radioactive decays, the IP exposure time required to reach a reasonable signal is comparable with the fast decay time of the IP fading. In this paper, we used the fading curve used in a recent paper, ${ }^{15}$ which avoids the aforementioned issues by exposing the IP to nanosecond pulses of photon radiation.

The fading has been measured for signal strength of the order of $10^{-3} \mathrm{PSL} / \mu \mathrm{m}^{2}$, which is equivalent to a PSL level of few units per pixel for an image scan resolution of $50 \mu \mathrm{m}$. The signal varies rapidly in the first 30 min after exposure, and then it decays slowly over several hours. Therefore, as a standard procedure, we have chosen to refer the response of IP to ions at $30 \mathrm{~min}$ after the exposure. This is done by rescaling the signal value according to the scanning time, using the following formula: ${ }^{15}$

$$
\operatorname{PSL}_{30}=\left(\frac{30}{t}\right)^{-0.161} \operatorname{PSL}(t),
$$

where PSL(t) represents the measured PSL signal obtained from the IP scanned " $t$ " min after the irradiation.

\section{Calibration curve}

The data points for the IP response curve were obtained from different shots and for carbon ions of different charge states, as shown in Fig. 4. The result shows that the response is independent of the initial ion charge state, consistently with the expectation that the stopping power depends only on the kinetic energy of the incident ion and its effective charge inside the matter. ${ }^{39,42-44}$ The data points were fitted with two empirical functions to obtain an analytical expression for the response,

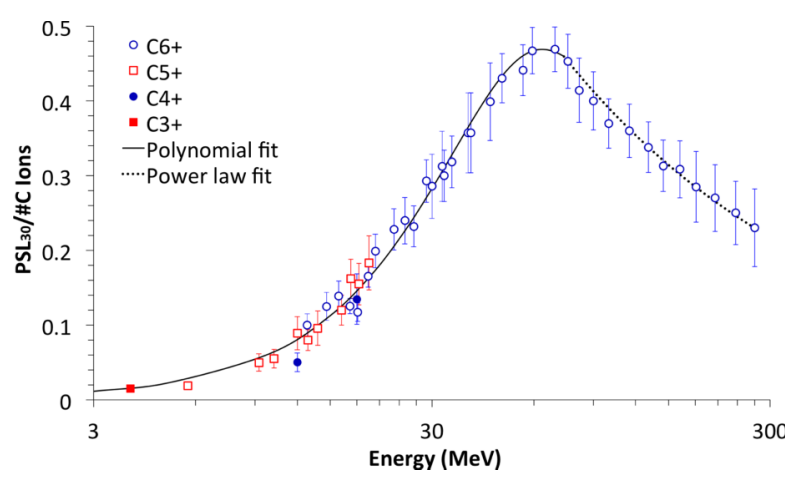

FIG. 4. The calibration curve relates the PSL signal to the number of carbon ions as function of the ion energy at the surface of IP. The curve is normalised to $30 \mathrm{~min}$ after the shot by using Eq. (2). 


$$
\mathrm{PSL}_{30} / \# \mathrm{C}=\left\{\begin{array}{ll}
\left(d+c \mathrm{E}+b \mathrm{E}^{2}+a \mathrm{E}^{3}\right) \mathrm{E} & 0 \leq \mathrm{E} \leq 73.6 \mathrm{MeV} \\
& a=+4.61 \times 10^{-8} \\
& b=-8.90 \times 10^{-6} \\
& c=+4.56 \times 10^{-4} \\
& d=+2.51 \times 10^{-3} \\
4.55 \mathrm{E}^{-0.533} & \mathrm{E}>73.6 \mathrm{MeV}
\end{array},\right.
$$

where $\mathrm{E}$ represents the carbon ion energy reaching the IP surface. The low energy part of the calibration curve is well reproduced by a 4th degree polynomial up to carbon energy of $73.6 \mathrm{MeV}$, while the calibration for higher energies can be represented by a power-law function. The analytical fit to the calibration data is shown in the Eq. (3). Although Fig. 4 shows the calibration for carbon ions with energies up to $270 \mathrm{MeV}$ (i.e., $22.5 \mathrm{MeV} /$ nucleon), Eq. (3) can be used to estimate the response to higher carbon ion energies considering that the deposition of energy scales as a power-law function when the stopping power of the ions in the active layer is far from the Bragg peak.

\section{Discussion}

The IP response to ions essentially depends on the fraction of energy deposited by the ions in the phosphor layer converted into excited metastable state. Although the shape of the IP response curve shown in Fig. 4 agrees qualitatively with the energy deposition profile of an ion in a layer of given thickness (Bragg's peak deposition profile), the energy stored in the IP cannot be directly related to the energy deposited by ions. In the case of very low ion energies (for instance, carbon energies below $10 \mathrm{MeV}$ ), the IP response becomes highly statistical in nature as the ion stopping range becomes comparable to or smaller than the grain size of the active material and the ions may only partly encounter active material grains during their transit. This would result in a weaker IP response for the low energy ions as a significant fraction of low energy ions would lose part of their energy while travelling through the "inactive" organic binder. However, the response of IP seems to be independent of the charge state of the incident ions, in agreement with stopping power theory, resulting in IP response which is virtually independent of the ionisation state of the ions.

\section{CONCLUSIONS}

We have presented the absolute response of the IP BASTR to carbon ions for energies up to $270 \mathrm{MeV}$, which can be reliably extrapolated to higher carbon energies using the empirical power-law fitting function. Moreover, the calibration confirms the independence of the IP response on the ion charge state which is particularly useful information for laserplasma interaction studies typically producing multi-species, multi-charge state ion beams. This calibration data allows quantitative use of this very sensitive detector with carbon ions.

\section{ACKNOWLEDGMENTS}

The authors acknowledge funding from EPSRC [No. EP/J002550/1-Career Acceleration Fellowship held by S.K., Nos. EP/L002221/1， EP/E035728/1， EP/K022415/1， EP/ J500094/1, EP/I029206/1, and EP/J003832/1], Laserlab Europe (No. EC-GA 284464), projects ELI (Grant No. CZ.1.05/ 1.1.00/483/02.0061), OPVK 3 (Grant No. CZ.1.07/2.3.00/20. 0279) and Invest Northern Ireland (POC-329). This work was also partly supported by the DFG GRK 1203 and SFB/TR18 programs. Authors also acknowledge the support of ESG, mechanical and target fabrication staff of the Central Laser Facility, STFC, UK.

${ }^{1}$ P. Bolton, M. Borghesi, C. Brenner, D. Carroll, C. De Martinis, A. Flacco, V. Floquet, J. Fuchs, P. Gallegos, D. Giove, J. S. Green, S. Green, B. Jones, D. Kirby, P. McKenna, D. Neely, F. Nuesslin, R. Prasad, S. Reinhardt, M. Roth, U. Schramm, G. Scott, S. Ter-Avetisyan, M. Tolley, G. Turchetti, and J. Wilkens, Phys. Med. 30, 255 (2014).

${ }^{2}$ See http://www.fujifilm.com for Fujifilm corporation.

${ }^{3}$ I. Paterson, R. Clarke, N. Woolsey, and G. Gregori, Meas. Sci. Technol. 19, 095301 (2008).

${ }^{4}$ P. Leblans, D. Vandenbroucke, and P. Willems, Materials 4, 1034 (2011).

${ }^{5}$ S. G. Gales and C. D. Bentley, Rev. Sci. Instrum. 75, 4001 (2004).

${ }^{6}$ K. A. Tanaka, T. Yabuuchi, T. Sato, R. Kodama, Y. Kitagawa, T. Takahashi, T. Ikeda, Y. Honda, and S. Okuda, Rev. Sci. Instrum. 76, 013507 (2005).

${ }^{7}$ N. Nakanii, K. Kondo, T. Yabuuchi, K. Tsuji, K. A. Tanaka, S. Suzuki, T. Asaka, K. Yanagida, H. Hanaki, T. Kobayashi, K. Makino, T. Yamane, S. Miyamoto, and K. Horikawa, Rev. Sci. Instrum. 79, 066102 (2008).

${ }^{8}$ H. Chen, N. L. Back, T. Bartal, F. N. Beg, D. C. Eder, A. J. Link, A. G. MacPhee, Y. Ping, P. M. Song, A. Throop, and L. Van Woerkom, Rev. Sci. Instrum. 79, 033301 (2008).

${ }^{9}$ A. L. Meadowcroft, C. D. Bentley, and E. N. Stott, Rev. Sci. Instrum. 79, 113102 (2008).

${ }^{10}$ A. Mancic, J. Fuchs, P. Antici, S. Gaillard, and P. Audebert, Rev. Sci. Instrum. 79, 073301 (2008).

${ }^{11}$ I. W. Choi, C. M. Kim, J. H. Sung, I. J. Kim, T. J. Yu, S. K. Lee, Y.-Y. Jin, K. H. Pae, N. Hafz, and J. Lee, Meas. Sci. Technol. 20, 115112 (2009).

${ }^{12}$ T. Bonnet, M. Comet, D. Denis-Petit, F. Gobet, F. Hannachi, M. Tarisien, M. Versteegen, and M. M. Aleonard, Rev. Sci. Instrum. 84, 013508 (2013).

${ }^{13}$ T. Bonnet, M. Comet, D. Denis-Petit, F. Gobet, F. Hannachi, M. Tarisien, M. Versteegen, and M. M. Alonard, Rev. Sci. Instrum. 84, 103510 (2013).

${ }^{14}$ C. G. Freeman, G. Fiksel, C. Stoeckl, N. Sinenian, M. J. Canfield, G. B. Graeper, A. T. Lombardo, C. R. Stillman, S. J. Padalino, C. Mileham, T. C. Sangster, and J. A. Frenje, Rev. Sci. Instrum. 82, 073301 (2011).

${ }^{15}$ A. Alejo, S. Kar, H. Ahmed, A. G. Krygier, D. Doria, R. Clarke, J. Fernandez, R. R. Freeman, J. Fuchs, A. Green, J. S. Green, D. Jung, A. Kleinschmidt, C. L. S. Lewis, J. T. Morrison, Z. Najmudin, H. Nakamura, G. Nersisyan, P. Norreys, M. Notley, M. Oliver, M. Roth, J. A. Ruiz, L. Vassura, M. Zepf, and M. Borghesi, Rev. Sci. Instrum. 85, 093303 (2014).

${ }^{16}$ T. Ohno, EPMA J. 4, 9 (2013).

${ }^{17} \mathrm{C}$. Schlaff et al., Radiat. Oncol. 9, 88 (2014).

${ }^{18}$ A. Macchi, M. Borghesi, and M. Passoni, Rev. Mod. Phys. 85, 751 (2013).

${ }^{19}$ S. Kar, K. Kakolee, B. Qiao, A. Macchi, M. Cerchez, D. Doria, M. Geissler, P. McKenna, D. Neely, J. Osterholz et al., Phys. Rev. Lett. 109, 185006 (2012).

${ }^{20}$ B. Hegelich, I. Pomerantz, L. Yin, H. Wu, D. Jung, B. Albright, D. Gautier, S. Letzring, S. Palaniyappan, R. Shah, K. Allinger, R. Hrlein, J. Schreiber, D. Habs, J. Blakeney, G. Dyer, L. Fuller, E. Gaul, E. Mccary, A. Meadows, C. Wang, Y. Ditmire, and J. Fernandez, New J. Phys. 15, 085015 (2013).

${ }^{21}$ M. Hegelich, S. Karsch, G. Pretzler, D. Habs, K. Witte, W. Guenther, M. Allen, A. Blazevic, J. Fuchs, J. C. Gauthier, M. Geissel, P. Audebert, T. Cowan, and M. Roth, Phys. Rev. Lett. 89, 085002 (2002).

${ }^{22}$ H. C. Kapteyn, A. Szoke, R. W. Falcone, and M. M. Murnane, Opt. Lett. 16, 490 (1991).

${ }^{23}$ B. Dromey, S. Kar, M. Zepf, and P. Foster, Rev. Sci. Instrum. 75, 645 (2004).

${ }^{24}$ C. Thaury, F. Quere, J. P. Geindre, A. Levy, T. Ceccotti, P. Monot, M. Bougeard, F. Reau, P. d'Oliveira, P. Audebert, R. Marjoribanks, and P. Martin, Nat. Phys. 3, 424 (2007).

${ }^{25}$ S. J. Gitomer, R. D. Jones, F. Begay, A. W. Ehler, J. F. Kephart, and R. Kristal, Phys. Fluids (1958-1988) 29, 2679 (1986). 
${ }^{26}$ S. C. Wilks, A. B. Langdon, T. E. Cowan, M. Roth, M. Singh, S. Hatchett, M. H. Key, D. Pennington, A. MacKinnon, and R. A. Snavely, Phys. Plasmas 8, 542 (2001).

${ }^{27}$ J. J. Thomson, Proc. R. Soc. London, Ser. A 89, 1 (1913).

${ }^{28}$ D. Gwynne, S. Kar, D. Doria, H. Ahmed, M. Cerchez, J. Fernandez, R. J. Gray, J. S. Green, F. Hanton, D. A. MacLellan, P. McKenna, Z. Najmudin, D. Neely, J. A. Ruiz, A. Schiavi, M. Streeter, M. Swantusch, O. Willi, M. Zepf, and M. Borghesi, Rev. Sci. Instrum. 85, 033304 (2014).

${ }^{29}$ R. Prasad, D. Doria, S. Ter-Avetisyan, P. Foster, K. Quinn, L. Romagnani, C. Brenner, J. Green, P. Gallegos, M. Streeter et al., Nucl. Instrum. Methods Phys. Res., Sect. A 623, 712 (2010).

${ }^{30}$ J. T. Morrison, M. Storm, E. Chowdhury, K. U. Akli, S. Feldman, C. Willis, R. L. Daskalova, T. Growden, P. Berger, T. Ditmire, L. Van Woerkom, and R. R. Freeman, Phys. Plasmas 19, 030707 (2012).

${ }^{31}$ See http://beamline.harima.-riken.jp/bl45xu/web_old/Info/BAS2500img Spec.pdf for Fujifilm IP scanner user manual.

${ }^{32}$ M. Kanasaki, T. Yamauchi, Y. Fukuda, H. Sakaki, T. Hori, M. Tampo, S. Kurashima, T. Kamiya, K. Oda, and K. Kondo, AIP Conf. Proc. 1465, 142 (2012).
${ }^{33}$ S. Kar, M. Borghesi, L. Romagnani, S. Takahashi, A. Zayats, V. Malka, S. Fritzler, and A. Schiavi, J. Appl. Phys. 101, 044510 (2007).

${ }^{34}$ N. Sinenian, M. J. Rosenberg, M. Manuel, S. C. McDuffee, D. T. Casey, A. B. Zylstra, H. G. Rinderknecht, M. Gatu Johnson, F. H. Sguin, J. A. Frenje, C. K. Li, and R. D. Petrasso, Rev. Sci. Instrum. 82, 103303 (2011).

${ }^{35}$ S. Gaillard, J. Fuchs, N. Renard-Le Galloudec, and T. E. Cowan, Rev. Sci. Instrum. 78, 013304 (2007).

${ }^{36} \mathrm{See} \mathrm{http}: / /$ scion-corporation.software.informer.com for Sdi corporation.

${ }^{37} \mathrm{See}$ http://rsbweb.nih.gov/ij/index.html for Imagej website.

${ }^{38}$ See http://www.scidi.com for S. D. I. Corporation.

${ }^{39}$ J. F. Ziegler, M. Ziegler, and J. Biersack, Nucl. Instrum. Methods Phys. Res., Sect. B 268, 1818 (2010).

${ }^{40}$ M. Thoms, Nucl. Instrum. Methods Phys. Res., Sect. A 378, 598 (1996).

${ }^{41}$ H. Ohuchi and A. Yamadera, Radiat. Meas. 35, 135 (2002).

${ }^{42}$ H. A. Bethe and J. Ashkin, Exp. Nucl. Phys. 1, 252 (1953).

${ }^{43}$ W. Brandt and M. Kitagawa, Phys. Rev. B 25, 5631 (1982).

${ }^{44}$ H. Betz, Rev. Mod. Phys. 44, 465 (1972). 